\title{
Aspiration biopsy of thoracic lesions
}

\author{
ANTHONY F. LALLI, BER NARD NAYLOR, AND \\ W A L TER M. WHITEHOUSE
}

From the Departments of Radiology and Pathology, University Hospital, Ann Arbor, Michigan, U.S.A.

\begin{abstract}
We have performed percutaneous needle aspiration biopsy of intrathoracic lesions under fluoroscopic control on 50 patients. Lesions as small as 1 to $1.5 \mathrm{~cm}$. in diameter were aspirated. Thirty of the 36 cases of malignant neoplasm were diagnosed by this method. Of the 14 nonneoplastic cases, granuloma was diagnosed in two, and in eight of the remaining 12 the clinical diagnosis was supported by the nature of the aspirated material. Pneumothoraces are to be expected as a complication of the method. Most will be asymptomatic; a small proportion will require treatment. There will also be the occasional haemoptysis. The procedure can be quickly performed and it is well tolerated by the patient. It causes less discomfort than bronchoscopy or scalene lymph node biopsy. The interpretation of the cytological material presents no undue difficulty to the pathologist experienced in conventional pulmonary exfoliative cytology.
\end{abstract}

Aspiration biopsy of pulmonary infiltrates, first practised in the nineteenth century, was primarily used to obtain material for culture in lobar pneumonia (Horder, 1909). The first recorded instance of aspiration biopsy of a pulmonary carcinoma occurred in 1886 (Menetrier, 1886). These early experiences, unassisted by radiographic localization of the lesions, were fairly successful in attaining their purpose; they also proved that needle aspiration was not necessarily complicated by empyema. Now the preciseness of modern radiological techniques makes it possible to aspirate small thoracic masses and infiltrates.

Nordenström in Sweden pioneered the use of small-bore needles and television $x$-ray fluoroscopy in the aspiration of thoracic masses (Nordenström, 1965a, b). He used this technique on more than 800 patients ; only in two did serious complications occur. Although small pneumothoraces were frequent, the procedure was considered safe enough to be performed on out-patients. We have adopted Nordenström's method and we report our results.

\section{METHOD}

Routine radiographic examinations of the chest are used to identify and localize the lesion. The patient is positioned on a horizontal $x$-ray table equipped with television fluoroscopy, preferably of the C-arm type. Fluoroscopy is used to localize the lesion, plan the approach, and mark the skin for puncture. The chest wall closest to the lesion is chosen, and the patient is positioned so that the direction of thrust $\stackrel{\square}{\Phi}$ of the needle toward the lesion is perpendicular to the floor and parallel to the $x$-ray beam. Occasionally $\overline{\bar{O}}$ the skeleton imposes an obstacle, making it necessary 3 to use an oblique course. Such a course is obligatory in lesions confined to the mediastinum.

The skin is cleansed, and the soft tissues are anaesthetized down to, and including, the pleura. $A$ 음 sterile No. 18 or No. 20 thin-walled spinal needle, complete with stylet and of sufficient length, usually 8 to 10 in. ( 20 to $25 \mathrm{~cm}$.), is advanced through the 3 anaesthetized area over the superior aspect of the adjacent rib and into the lung. Occasionally a jabbing motion is necessary to penetrate the mass. The depth

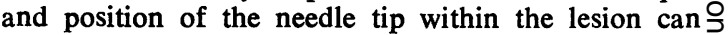
be determined by rotating the $C$-arm or the patient. $D$ Exposure of the operator's hands to irradiation is minimized by using fluoroscopy only after each $\mathrm{N}$ change of position of the needle has been made. A $\mathcal{O}$ pulmonary nodule can be made to move when the $N$ needle is in it or very near to it.

When the point of the needle seems to be in the lesion, the stylet is partly withdrawn and the needle is rotated to loosen material. Aspiration is carried outeo with a syringe having a close-fitting plunger. The $\frac{\bar{D}}{\mathscr{C}}$ syringe and attached needle are then withdrawn às a? unit, maintaining a slight negative pressure in the $\square$ syringe to avoid losing the specimen. Frequently, the $\overline{0}$ specimen is quite small and does not appear in the $\mathbb{D}$ syringe but is contained entirely within the needle.

The specimen is blown out of the needle on to slides, and smeared and fixed in $95 \%$ ethyl alcohol 
before the slightest trace of drying occurs. The syringe and needle are then rinsed in normal saline. In the laboratory the smears are stained by the Papanicolaou method. The saline washing is centrifuged, the supernatant is poured off, and to the centrifugate are added a few drops of plasma followed by a few drops of aqueous thrombin solution. Within a few seconds a small clot forms. This is cut into smaller pieces, fixed in formalin, and processed like small pieces of tissue. In addition to the routine haematoxylin and eosin stained sections, a variety of special stains can be applied to this sectioned material.

One can repeat the examination on the same day or on subsequent days. After the examination, a chest radiograph is obtained to see if pneumothorax is present, and further films are obtained if, during the next few hours, the patient becomes breathless. Pneumothoraces of significant size usually become apparent during the early post-biopsy period, and if the patient has pulmonary emphysema they can be expected.

MATERIAL

We have used aspiration biopsy on 50 patients with thoracic lesions. One lesion was mediastinal, four were in the chest wall, and the rest were within the lung. The patients' ages ranged from 25 to 81 years, and all were in-patients at the time of the examination. In most instances sputum cytology, scalene lymph node biopsy, and bronchoscopy had been used and had been unsuccessful in making a diagnosis.

The primary criterion for the performance of this examination is the ability to identify the lesion fluoroscopically. We have successfully taken biopsy specimens from lesions as small as $1 \mathrm{~cm}$. in diameter; we shall attempt to aspirate smaller lesions. Lesions within the lung, but near the chest wall or diaphragm, can be difficult to find unless they are plainly visible and approachable by a perpendicular thrust of the needle.

There are various circumstances which present indications against this type of needle biopsy; however, each case has to be individually evaluated. It is important to select only co-operative patients, for it is not desirable to use general anaesthesia. A lesion suspected of being a cavernous haemangioma or an arteriovenous fistula should not be needled. Patients who are receiving anticoagulants or who have pulmonary hypertension may also be unsuitable for a biopsy, though generally the presence of emphysema is not a contraindication.

The biopsy was repeated once in three patients, thereby enabling diagnostic material to be obtained. We should have obtained better results had repeated biopsies been possible in each patient whose first biopsy gave negative results.

\section{RESULTS}

Abnormal material was obtained from 43 $(86 \%)$ of the 50 patients. In 40 cases $(80 \%)$ it was possible, from this material, to make a definite diagnosis of malignant neoplasm or granuloma, or to support another clinical diagnosis. Of the 36 cases of malignant neoplasm, $30(82 \%)$ were diagnosed by this method, and in two cases inflammatory material was identified but malignant cells were not seen. Of the 14 non-neoplastic cases the diagnosis of granuloma was made in two, and in eight of the remaining 12 the clinical diagnosis was supported by the nature of the aspirated material.

The malignant neoplasms identified by this method were: adenocarcinoma (13 cases), squamous-cell carcinoma (8 cases), undifferentiated carcinoma (5 cases), malignant lymphoma ( 2 cases), malignant melanoma (1 case), and myeloma (1 case).

\section{COMPLICATIONS}

Five of the 50 patients had small haemoptyses lasting not more than one hour. Twenty-two had pneumothoraces, three of which had to be treated with chest tube aspiration for $\mathbf{2 4}$ or $\mathbf{4 8}$ hours. Two of these, occurring in elderly men with bullous emphysema, were expected. Most of the pneumothoraces were quite small, resolving quickly and not producing any symptoms. A few patients developed subcutaneous emphysema, but this was of no clinical significance. As yet there has been no instance of haemothorax nor has there been any air embolism.

\section{DISCUSSION}

Our experience with percutaneous, fluoroscopically controlled aspiration biopsy of thoracic lesions has convinced us that it is a useful and reliable diagnostic procedure. In our hands, as in the hands of others (Martin and Stewart, 1936; Woolf, 1954 ; Dahlgren and Nordenström, 1966), the mortality has been nil and the morbidity has been acceptable. Furthermore, patients are only slightly discomforted by this quickly performed procedure, certainly much less than by bronchoscopy or scalene lymph node biopsy.

In most of our patients sustained attempts to reach a diagnosis had been fruitless before aspiration biopsy, and in fact most of the patients were referred to us because other methods had failed 
to provide a definite diagnosis. The value of aspiration biopsy in our patients was demonstrated by the fact that this procedure provided a definite diagnosis, or materially contributed to the diagnosis, in 32 out of 39 patients with negative sputum cytology, in 15 out of 20 with negative scalene node biopsies, and in 20 out of 24 who had undergone bronchoscopy with inconclusive results. For many of the patients, several weeks in hospital and considerable expense would have been avoided had aspiration biopsy been resorted to earlier. But, as with any new or reintroduced procedure, resistance has to be overcome and the confidence of the clinicians has to be won.

Inevitably, the success of the method depends on the ability of the pathologist to interpret the aspirated material. For a pathologist experienced in conventional pulmonary exfoliative cytology the material obtained by aspiration biopsy does not present any undue difficulty. In fact, it is usually easier and quicker to make the diagnosis of cancer from the aspirated biopsy material than from sputum or bronchial secretions, simply because it contains such large numbers of neoplastic cells. The neoplastic cells in smears of this type of aspirated material are, perhaps, slightly more difficult to classify. This difficulty may be overcome if sufficient neoplasm is aspirated so that sections can be prepared from the centrifugate of the saline washings.

The aspiration biopsy samples only a small volume of tissue and, although normal tissue or non-diagnostic tissue may be obtained at the first attempt, the surrounding tissue may yield diagnostic material. Bearing this in mind, there is no doubt that one could achieve a high diagnostic accuracy by keeping the patient in the radiology department and repeating the biopsies until the pathologist is able to give a definite diagnosis. Such an arrangement would require all biopsies to be scheduled at a time which could be reserved by both the radiologist and pathologist. Also, the pathologist would have to use a rapid staining method to reduce the delay between obtaining the material and reporting on it.

Whether or not such an arrangement is used (and, incidentally, we did not use it), it is most desirable to have a close liaison between the pathologist and the radiologist in order to decide whether a negative finding should be regarded as significant. In this situation, one has to keep in mind the most probable diagnosis on the basis of clinical and radiographic findings. It is regrettable, but generally true, that the only significant finding on aspiration biopsy of a patient suspected of harbouring a malignant neoplasm is the presence $\frac{}{5}$ of neoplastic cells. Even the finding of inflamma- $\frac{\bar{\sigma}}{\bar{N}}$ tory material, with the exception of a granulo- $\frac{\widehat{\phi}}{\delta}$ matous reaction, might have little significance, $\Omega$ since malignant neoplasms are commonly accompanied by inflammation.

The frequently recurring objection to needle $\overrightarrow{\vec{A}}$ aspiration of intrathoracic lesions is that neoplasm $\stackrel{\sigma}{\sigma}$ or infection will be spread by the needle as it is $\overrightarrow{\vec{*}}$ withdrawn (Ochsner and DeBakey, 1941). No evidence of such spread has occurred in Dahlgren $N$ and Nordenström's (1966) series, now extending or over five years. Our experience gained during $\stackrel{p}{\nexists}$ a nine-month period is not enough to be used as a basis for debating this point. However, $\stackrel{ }{\rightarrow}$ it is just possible that there may be an increased $\vec{c}$ risk of blood-borne metastases.

We think the use of a small-bore needle may $\frac{\vec{D}}{\vec{D}}$ help to minimize the threat of spread of disease $\frac{3}{0}$ along the needle tract. Several hundred cases have been reported in which Franklin or Vim-Silver- $\vec{\varphi}$ man needles, or needles of even greater diameter, $\stackrel{\odot}{V}$ were used, and in none of these cases was there evidence of spread of neoplasm or infection along the needle tract (Martin and Stewart, 1936; Craver and Binkley, 1939 ; Aronovitch, Chartier, Kahana, Meakins, and Groszman, 1963 ; Krum- $\stackrel{\odot}{\mathcal{D}}$ holz, Manfredi, Weg, and Rosenbaum, 1966).

It is relevant to recall that, for thoracentesis, $\stackrel{\overline{\bar{O}}}{\bar{z}}$ one uses needles of large diameter, and yet it is rare to find a neoplasm or infection spreading along the needle tract, though such spread may occur from carcinomatous involvement of the $\vec{\partial}$ pleura. Diffuse malignant mesotheliomata are recognized to have this tendency to spread along $\underset{\dot{\sigma}}{x}$ needle tracts, a tendency which can be attributed 3 to some inherent biological property of the neoplasm (Rose, Palmer, and Lougheed, 1955; Godwin, 1957).

Any actual or theoretical dangers of needle aspiration biopsy should be weighed against the dangers inherent in thoracotomy and the possibility of spontaneous dissemination of neoplasm while $\overparen{N}$ other diagnostic procedures, taking days or weeks, N are being carried out. The ease and rapidity with $\underset{\mathrm{N}}{\mathrm{N}}$ which aspiration biopsy can give diagnostic $O$ material (the entire process with a final diagnosis often takes less than one hour), and the fact that $\stackrel{\odot}{\oplus}$ the procedure can, in some instances, be per- $\stackrel{D}{\rightarrow}$ formed on out-patients must also be taken into 7 account when planning the approach to the 0 diagnosis of intrathoracic lesions. We feel that $\underset{\mathbb{D}}{\stackrel{D}{ }}$ there are times when the potential benefits of $\frac{O}{\mathbb{D}}$ needle aspiration outweigh its dangers. 


\section{REFERENCES}

Aronovitch, M., Chartier, J., Kahana, L. M., Meakins, J. F., and Groszman, M. (1963). Needle biopsy as an aid to the precise diagnosis of intrathoracic disease. Canad. med. Ass. J., 88, 120.

Craver, L. F., and Binkley, J. S. (1939). Aspiration biopsy of tumors of the lung. $J$. thorac. Surg., 8, 436.

Dahlgren, S., and Nordenström, B. (1966). Transthoracic Needle Biopsy. Almqvist and Wiksell, Stockholm.

Godwin, M. C. (1957). Diffuse mesotheliomas with comment on their relation to localized fibrous mesotheliomas. Cancer (Philad.), 10 , 298.

Horder, T. J. (1909). Lung puncture: a new application of clinical pathology. Lancet, 2, 1345.

Krumbolz, R. A., Manfredi, F., Weg, J. G., and Rosenbaum, D. (1966). Needle biopsy of the lung. Ann. intern. Med.. 65, 293.
Martin, H. E., and Stewart, F. W. (1936). The advantages and limitations of aspiration biopsy. Amer. J. Roentgenol., 35, 245.

Menetrier, P. (1886). Cancer primitif du poumon. Bull. Soc. anat. Paris, 11, 643.

Nordenström, B. (1965a). Therapeutic roentgenology. Acta radiol. 3,115 .

- (1965b). A new technique for transthoracic biopsy of lung changes. Brit. J. Radiol., 38, 550.

Ochsner, A., and DeBakey, M. (1941). Primary carcinoma of the lung. New Orleans med. surg. J., 93, 387.

Rose, R. G., Palmer, J. D., and Lougheed, M. N. (1955). Treatment of peritoneal mesothelioma with radioactive colloidal gold Cancer (Philad.), 8, 478.

Woolf, C. R. (1954). Applications of aspiration lung biopsy with a review of the literature. Dis. Chest, 25, 286. 